\title{
DETERMINATION OF INELASTIC MEAN FREE PATH OF ELECTRONS IN NOBLE METALS
}

\author{
W. Doliński, S. Mróz, J. Palczyński \\ Institute of Experimental Physics, University of Wrocław \\ Cybulskiego 36, 50-205 Wrocław, Poland \\ B. Gruzza, P. Bondot and A. Porte \\ Laboratoire de Physique des Milieux Condensés, CNRS 796 \\ Université Blaise Pascal-Clermont Ferrand II, 63177 Aubiere Cedex, France \\ (Received May 22, 1991; in revised form September 9, 1991)
}

\begin{abstract}
The experimental values of Inelastic Mean Free Path ( $\lambda_{\text {IMFP }}$ ) of electrons in noble metals $(\mathrm{Ag}, \mathrm{Au}, \mathrm{Cu})$ are determined in the electron energy range $150-2000 \mathrm{eV}$. The method used consists of the measurements and theoretical calculations of the coefficient of elastic backscattering of electrons frcm a solid surface $\eta_{e}$. The obtained values of $\lambda_{\text {IMFP }}$ are compared with the data a vailable in the literature.
\end{abstract}

PACS numbers: 72.10.Fk, 79.20.Fv

\section{Introduction}

The value of the probability that the photoelectron or Auger electron created in the sample leaves the sample without energy loss is an important factor in the mathematical formalism of quantitative XPS or AES. Such an electron transport is characterized by several different terms in the literature i.e. inelastic mean free path (IMFP), escape depth (ED), attenuation length (AL) and also an information depth (ID). It should be pointed here that each of these terms has a separate meaning (see Ref. [1-4]). Also their values can be different. For example Powell [5] and Jablon'ski et al. [3, 6-9] have shown that the difference between IMFP and $\mathrm{AL}$ can be equal to $30 \%$ or even more. This considerable difference originates among others from different methods of the $\mathrm{AL}$ and IMFP determination. Namely, the $A L$ values have been determined from the overlayer experiments and with an assumption that the elastic scattering of the electrons can be neglected. Based on the results of such experiments performed for many overlayer-substrate systems and different electron energies Seah and Dench [10] proposed the formulae for AL value calculations for elements and compounds. IMFP values (as being inversely 
related to the total inelastic scattering cross-section) were mainly obtained basing on the calculation of the total probability of inelastic scattering of electrons in solids (see Ref. [11-15]). In 1985 Jabłoński [16] proposed an experimental method by which IMFP value for given electron energy and an element can be determined from the measurements and theoretical calculations of the coefficient of elastic electron backscattering from the solid. This method was used previously $[17,18]$ for the determination of electron IMFP values for $\mathrm{Ag}$ and $\mathrm{Cu}$ and a few values of electron energies. In this paper we present the results of IMFP measurements for noble metals $\mathrm{Ag}, \mathrm{Au}, \mathrm{Cu}$ ) in the electron energy range 150-2000 eV (including characteristic Auger energies).

\section{Description of the method}

In the method proposed by Jabłoński [16] IMFP values $\left(\lambda_{\text {IMFP }}\right)$ are obtained from the comparison of the elastic electron backscattering coefficient $\eta_{\mathrm{e}}$ measured for a given electron energy and a given sample with the value of this coefficient calculated theoretically for an assumed value of $\lambda_{\mathrm{IMFP}}$. The measured $\eta_{\mathrm{e}}$ value is defined as follows:

$$
\eta_{\mathrm{e}}=\frac{I_{\mathrm{el}}}{I_{\mathrm{p}}}
$$

where $I_{\mathrm{el}}$ - the current of electrons elastically backscattered from the sample, $I_{\mathrm{p}}$ - primary electron beam current. The theoretical $\eta_{\mathrm{e}}$ value is obtained for a given sample, electron energy and an assumed $\lambda_{\text {IMFP }}$ value by computer simulation of electron trajectories in the sample. The computer randomizes the electron paths in a solid between successive elastic collisions as well as polar and azimuthal angles of each elastic scattering. Such operations are repeated until an electron leaves the sample or its total path will be larger than an assumed multiplicity of $\lambda_{\text {IMFP }}$. In a first case the contribution of a single electron to the total current of electrons backscattered elastically is

$$
\mathrm{d} I_{\mathrm{el}}=\exp \left(\frac{-\sum_{\mathrm{i}} z_{i}}{\lambda_{\mathrm{IMFP}}}\right)
$$

where $\sum_{\mathrm{i}} z_{i}-$ total path of an electron in the sample, $\lambda_{\mathrm{IMFP}}-$ assumed value of $\lambda_{\text {IMFP }}$, if $\sum_{\mathrm{i}} z_{i}>k^{*} \lambda_{\mathrm{IMFP}} \Rightarrow \mathrm{d} I_{\mathrm{el}} \approx 0$ for $k \geq 11$.

If one performs such simulations for the same electron energy and several assumed $\lambda_{\text {IMFP }}$ values one can obtain the theoretical dependence of $\eta_{e}$ on $\lambda_{\text {IMFP }}$. From such dependence the experimental $\lambda_{\text {IMFP }}$ value can be found as related to the $\eta_{\mathrm{e}}$ measured experimentally. The procedure of $\eta_{\mathrm{e}}$ measurements was presented previously [17]. Some changes were made, however, in the theoretical calculations of $\eta_{\mathrm{e}}$. So, the used method of calculations is shortly described below.

\subsection{Calculations of $\eta_{\mathrm{e}}$}

The problem of elastic scattering of electrons can be solved within the nonrelativistic partial wave expansion method [19]. Considering the process of elastic scattering one can precise that: 
- the incident electron is represented by a plane wave of unit amplitude traveling in the $z$ direction:

$\Psi_{\mathrm{i}}=\exp (\mathrm{i} k z)$;

- the scattered wave $\Psi_{\mathrm{s}}$ travels out radially from the scattering atom with an amplitude which is a function of the scattering angle $\theta$ as well as the distance $r$;

- the sum of these two waves

$\Psi(r, \theta)=\Psi_{\mathrm{i}}+\Psi_{\mathrm{s}}$

is a solution of the Schrödinger equation:

$\nabla^{2} \Psi+\left(k^{2}-2 m V(r) / \hbar^{2}\right) \Psi=0$

in which $k=2 \pi / \lambda, \lambda$ is the associated de Broglie wavelength and the spherical potential $V(r)$ can be expressed as the Thomas-Fermi-Dirac one [20, 21].

The following formulae are obtained:

$$
\begin{aligned}
& \Psi_{\mathrm{i}}(r, \theta)=C_{\mathrm{i}} \sum_{n \approx 0}^{\infty} P_{n}(\cos \theta) g_{n}(r) / r \\
& \Psi_{\mathrm{s}}(r, \theta)=C_{\mathrm{s}} \sum_{n=0} P_{n}(\cos \theta) G_{n}(r) / r
\end{aligned}
$$

where the $g_{n}$ 's and $\mathrm{G}_{n}$ 's are solutions of the differential equations:

$$
\begin{aligned}
& \mathrm{d} g_{n}^{2} / \mathrm{d} r^{2}+\left(k^{2}-n(n+1) / r^{2}\right) g_{n}=0, \\
& \mathrm{~d} G_{n}^{2} / \mathrm{d} r^{2}+\left(k^{2}-2 m V(r) / \hbar^{2}-n(n+1) / r^{2}\right) G_{n}=0 .
\end{aligned}
$$

The number of electrons scattered elastically per unit solid angle is given by the differential scattering cross-section:

$$
\mathrm{d} \sigma / \mathrm{d} \Omega=|f(\theta)|^{2}=A^{2}+B^{2}
$$

where

$$
\begin{aligned}
& A=\left(2 k^{-1}\right) \sum_{n=0}^{\infty}(2 n+1)\left(\cos 2 \delta_{n}-1\right) P_{n}(\cos \theta) \\
& B=\left(2 k^{-1}\right) \sum_{n=0}^{\infty}(2 n+1) \sin 2 \delta_{n} \cdot P_{n}(\cos \theta)
\end{aligned}
$$

$P_{n}(\cos \theta)$ are the Legendre coefficients and $\delta_{n}$ the phase shifts between the asymptotic sinusoidal expressions of $g_{n}(r)$ and $G_{n}(r)$.

The value of $\delta_{n}$ can be obtained when $V(r)$ is becoming insignificant [22]. The curves $g_{n}(r)$ and $G_{n}(r)$ are not yet sinusoidal but as they are solutions of the same differential equation the phase remains constant. The time of computer calculation is then well limited.

In our process of calculation only numerical methods are used. Equations (3) and (4) are solved simultaneously, the procedure is based on:

- an expanded solution into a series for the known initial conditions;

- the high accuracy method devised by W.E. Milne [23] with predicting and checking the solutions of the differential equations.

Using a step of iteration equal to $5 \times 10^{-3} \AA$, all our results have been obtained with a DPX 2000 Bull computer with the 68020 Motorola microprocessor. 


\section{Experimental}

Values of $\eta_{\mathrm{e}}$ were measured for three noble metals $(\mathrm{Ag}, \mathrm{Au}, \mathrm{Cu})$ in the electron energy range 150-2000 eV. As an electron energy analyzer we used 4-grid RFA with an acceptance angle $4-44^{\circ}$ and electron energy resolution equal to $0.6 \%$. The clean, polycrystalline $\mathrm{Ag}, \mathrm{Au}, \mathrm{Cu}$ samples (purity $99.99 \%$ ) were prepared in the form of foils $7 \times 14 \mathrm{~mm}, 0.4 \mathrm{~mm}$ thick. Their surfaces were cleaned with potassium ion bombardment $\left(E_{\mathrm{K}+}=400 \mathrm{eV}\right)$ and heated to $\approx 920 \mathrm{~K}$. The surface purity was controlled with AES. The pressure during the measurements did not exceed $4 \times 10^{-10}$ torr.

\section{Results, discussion and conclusions}

The results of $\eta_{\mathrm{e}}$ measurements for $\mathrm{Ag}, \mathrm{Au}$, and $\mathrm{Cu}$ are presented in Fig. 1 (solid lines), together with $\eta_{\mathrm{e}}$ values obtained by Schmid et al. [23] (dashed lines) who also used the RFA analyzer. As it is visible the shapes of $\eta_{\mathrm{e}}=f(E)$ dependencies are quite similar, but the $\eta_{\mathrm{e}}$ values are different. This difference originates

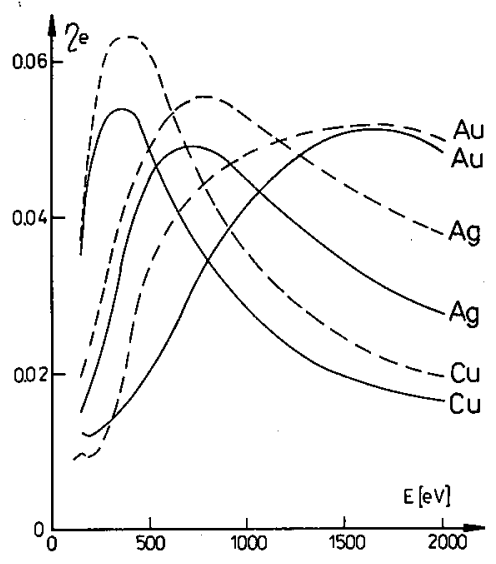

Fig. 1. The comparison of the measured $\eta_{e}$ values with those obtained by Schmid et al. [24] (solid lines - our results, dashed lines — the results from Ref. [24]).

probably from different RFA acceptance angles $\left(4-44^{\circ}\right.$ in our measurements, $6-52^{\circ}$ in Ref. [23]). The calculated values of the amplitudes $f(\theta)$ are in good agreement with those published previously. For example in the case of electrons scattered by $\mathrm{Ag}$ atoms one can see:

- in Fig. 2 a comparison with the calculations of M. Fink and J. Ingram [25] obtained using the relativistic Hartree-Fock-Slater potential;

- in Fig. 3 our results are compared with those by Jabłoński [26] who used the same form of the potential $V(r)$ but with another mode of the mathematical process to get the phase $\delta_{n}$.

The theoretical values of $\eta_{\mathrm{e}}$ were obtained for electron energies from the range 150-2000 eV and for 6 assumed values of IMFP for each value of electron 

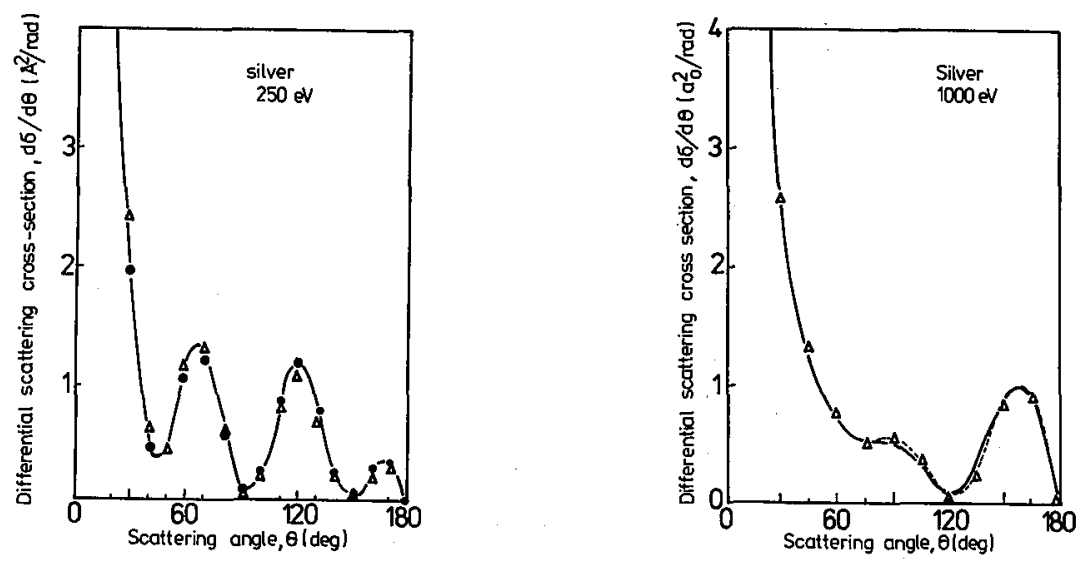

Fig. 2. The dependence of the differential cross-section for elastic scattering $2 \pi f^{2}(\theta)$ $\times \sin \theta \mathrm{d} \theta$ for electron energy equal to $250 \mathrm{eV}$ ( - Ref: [25], $\Delta-$ our results).

Fig. 3. The dependence of the differential cross-section for elastic scattering $2 \pi f^{2}(\theta)$ $x \sin \theta \mathrm{d} \theta$ for electron energy equal to $1000 \mathrm{eV}$ (solid and dashed lines - the nonrelativistic and the relativistic PWEM taken from Ref. [26]; $\triangle$ - our results).

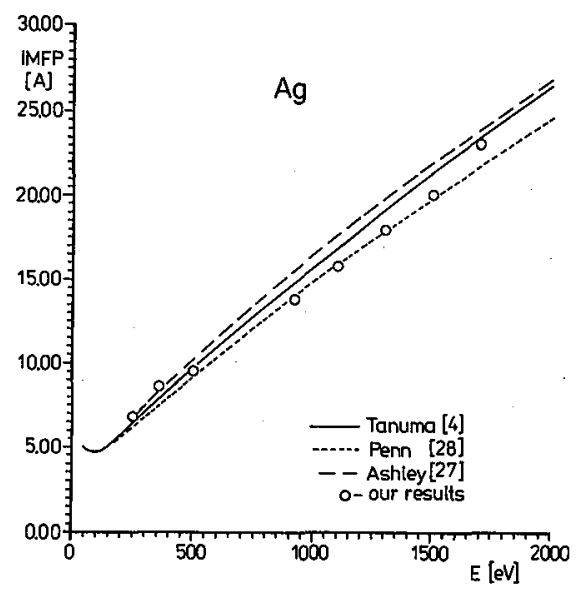

Fig. 4. Values of $\lambda_{\text {IMFP }}$ as a function of electron energy determined for Ag.

energy using one million electrons impinging the surface. The determined $\lambda_{\text {IMFP }}$ values compared with those recommended by Ashley and Tung [27], Penn [28] and calculated recently by Tanuma et al. [4] are presented in Figs. 4-6 as a function of electron energies. An excellent agreement of our $\lambda_{\text {IMFP }}$ with those calculated theoretically is observed for silver sample. For gold and copper they differ from the theoretical values by about $10-20 \%$ or even more especially in the low energy region. However, one can notice a good agreement with Ashley and Tung [27] results for the gold sample in the high energy region. Our approach to $\lambda_{\text {IMFP }}$ 


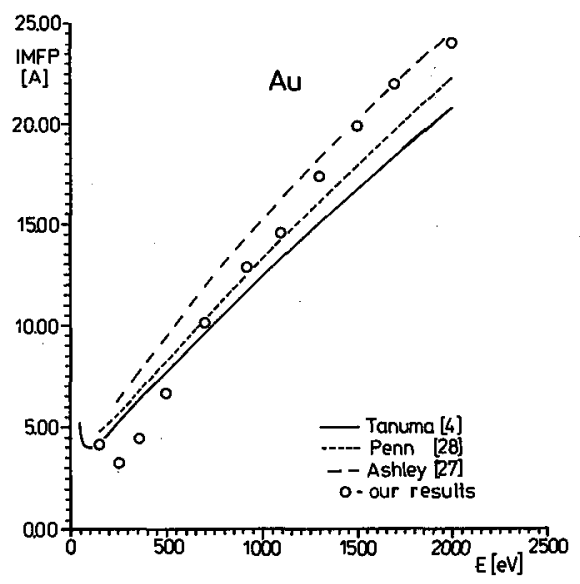

Fig. 5. Values of $\lambda_{\text {IMFP }}$ as a function of electron energy determined for Au.

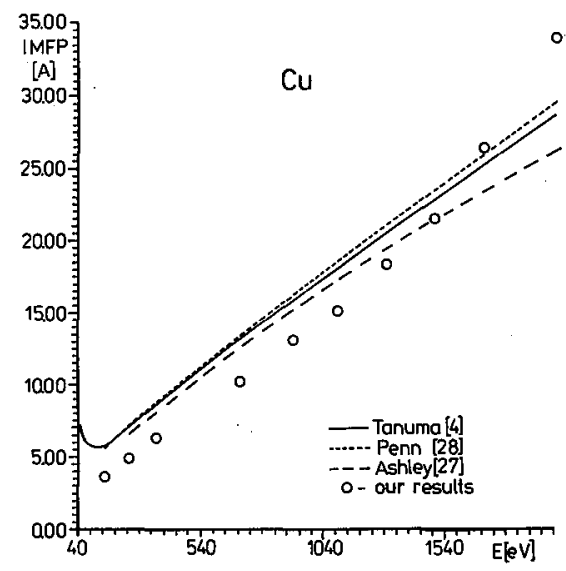

Fig. 6. Values of $\lambda_{\text {IMFP }}$ as a function of electron energy determined for $\mathrm{Cu}$.

determination is completely different from those used in theoretical calculations $[4,27,28]$. So, nothing can be said about the possible reasons of the observed discrepancies in the results obtained for copper and gold. Additionally it is difficult to estimate the accuracy of our method. $\eta_{\mathrm{e}}$ was measured with RMS $<5 \%$, the precision of computer Monte-Carlo simulations was not worse than $1 \%$, but the results depend also on the accuracy of $f(\theta)$ theoretical calculations.

\section{Acknowledgements}

This work was supported by University of Wrocław (grant 10.10/S/IFD/91). 


\section{References}

[1] ASTM Standard Definitions of Terms Relating to Surface Analysis, Surf. Interface Anal. 10, 48 (1987).

[2] C.J. Powell, J. Electron Spectrosc. Relat. Phenom. 47, 197 (1988).

[3] A. Jabloński, H. Ebel, Surf. Interface Anal. 11, 627 (1988).

[4] S. Tanuma, C.J. Powell, D.R. Penn, J. Electron Spectrosc. Relat. Phenom. 52, 285 (1990).

[5] C.J. Powell, J. Vac. Sci. Technol. A3, 1338 (1985); 4, 1532 (1986).

[6] A. Jabłoniski, Surf. Sci. 188, 164 (1987).

[7] A. Jablonski, M. Krawczyk, B. Lesiak, J. Electron Spectrosc. Relat. Phenom. 46, 131 (1988).

[8] A. Jabłoński, B. Lesiak, II. Ebel, M.F. Ebel, Surf. Interface Anal. 12, 87 (1988).

[9] A. Jabłoński, Surf. Interface Anal. 14, 659 (1989).

[10] M.P. Seah, W.A. Dench, Surf. Interface Anal. 1, 2 (1979).

[11] C.J. Powell, Surf. Interface Anal. 7, 263 (1985).

[12] D.R. Penn, J. Electron Spectrosc. Relat. Phenom. 9, 29 (1976).

[13] J.C. Ashley, C.J. Tung, R.H. Ritchie, Surf. Sci. 81, 409 (1979).

[14] C.J. Tung, J.C. Ashley, R.H. Ritchie, Surf. Sci. 81, 427 (1979).

[15] J. Szajman, J. Liesegang, J.G. Jenkin, R.C.G. Leckey, J. Electron Spectrosc. Relat. Phenom. 23, 97 (1981).

[16] A. Jabłoński, Surf. Sci. 151, 166 (1985).

[17] W. Doliński, H. Nowicki, S. Mróz, Surf. Interface Anal. 11, 229 (1988).

[18] W. Doliński, S. Mróz, M. Zagórski,Surf. Sci. 200, 361 (1988).

[19] H. Faxen, J. Holtsmark, Z. Phys. 45, 307 (1927).

[20] R.A. Bonham, T.G. Strand, J. Chem. Phys. 39, 2200 (1963).

[21] S. Ichimura, R. Shimizu, Surf. Sci. 112, 386 (1981).

[22] F. Calogero, Variable Phase Approach to Potential Scaltering, Academic Press, New York 1967.

[23] W.E. Milne, American Mathematical Monthly 33, 455 (1926).

[24] R. Schmid, K.H. Gaukler, H. Seiler, Scanning Electron Microscopy-1983 II (1983), p. 501.

[25] M. Fink, D. Ingram, At. Data Nucl. Data Tables 4, 129 (1972).

[26] A. Jabloński, Surf. Interface Anal. 14, 659 (1989).

[27] J.C. Ashley, C.J. Tung, Surf. Interface Anal. 4, 52 (1982).

[28] D.R. Penn, Phys. Rev. B 35, 482 (1987). 